Production, Manufacturing and Logistics

\title{
Transportation pricing of a truckload carrier
}

\author{
Ayşegül Toptal ${ }^{\mathrm{a}, *}$, Safa Onur Bingöl ${ }^{\mathrm{b}}$ \\ ${ }^{a}$ Industrial Engineering Department, Bilkent University, Ankara, 06800, Turkey

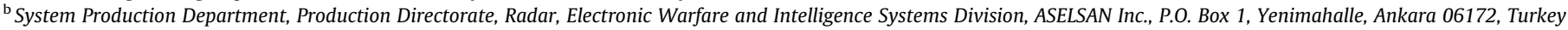

\section{A R T I C L E I N F O}

Article history:

Received 24 December 2009

Accepted 3 May 2011

Available online 11 May 2011

\section{Keywords:}

Transportation pricing

Supply chain management

Truckload carrier

Less than truckload carrier

Integrated inventory/transportation

\begin{abstract}
A B S T R A C T
Freight transportation is a major component of logistical operations. Due to the increase in global trade, fierce competition among shippers and raising concerns about energy, companies are putting more emphasis on effective management and usage of transportation services. This paper studies the transportation pricing problem of a truckload carrier in a setting that consists of a retailer, a truckload carrier and a less than truckload carrier. In this setting, the truckload carrier makes his/her pricing decision based on previous knowledge on the less than truckload carrier's price schedule and the retailer's ordering behavior. The retailer then makes a determination of his/her order quantity through an integrated model that explicitly considers the transportation alternatives, and the related costs (i.e., bimodal transportation costs) and capacities. In the paper, the retailer's replenishment problem and the truckload carrier's pricing problem are modeled and solved based on a detailed analysis. Numerical evidence shows that the truckload carrier may increase his/her gainings significantly through better pricing and there is further opportunity of savings if the truckload carrier and the retailer coordinate their decisions.
\end{abstract}

(c) 2011 Elsevier B.V. All rights reserved.

\section{Introduction}

Freight transportation is a significant component of the global economy. United States (US) and European Union (EU) hold the first two places in the world for their freight transportation expenditures. Freight shipping costs have been over $10 \%$ of the US Gross Domestic Product (GDP) in 2007 (http://www.bts.gov). In EU countries, volume of the freight transportation is expected to increase by $50 \%$ from 2000 to 2020 , while the EU GDP is expected to grow by $52 \%$ in the same period (http://ec.europa.eu/transport/strategies/2006_keep_europe_moving_en.htm). In light of the above statistics, minimization of transportation costs presents an important opportunity for companies to improve their profits. Revenue management is another tool that can be used by the freight carriers for this purpose. However, there is limited research suggesting pricing policies for transportation companies. With the increasing oil prices, uncertainty in the market and the raising competition among transporter companies, this topic has become well worth to study. The objectives of this paper are to suggest methods for truckload carriers to make their pricing decisions and to quantify the savings that can be achieved through these decisions.

With the above objectives in mind, we consider a system that consists of a retailer, a truckload (TL) carrier and a less than

\footnotetext{
* Corresponding author. Tel.: +90 3122901702.

E-mail addresses: toptal@bilkent.edu.tr (A. Toptal), sobingol@mst.aselsan.com.tr (S.O. Bingöl).
}

truckload (LTL) carrier. The retailer purchases the services of these carriers for inbound transportation and makes his/her replenishment decisions considering the related transportation costs and capacities. The TL carrier charges a fixed price per truck regardless of whether the truck is fully or partially loaded. The LTL carrier charges in proportion to the number of units shipped. Both the carriers announce their pricing schedules before the retailer makes his/her replenishment decision.

In this paper, we focus on the pricing problem of the TL carrier. We consider a case where the TL carrier knows the LTL carrier's tariff schedule at the time of his/her pricing decision. This may be possible if the LTL carrier uses a base rate which is industry standard, or simply because he/she has preannounced freight rates. The TL carrier also has full information about the retailer or his/her ordering behavior. In this setting, we study the TL carrier's problem of finding the price to charge for a single truck with the objective of maximizing the total revenues. Our focus on the TL carrier's pricing problem given the LTL carrier's pricing decision is motivated by contract logistics involving 3PL practices. In our experience with the freight industry in Turkey, we have observed that there are truckload carriers with the following common characteristics: their business with a majority of the customers are in the form of arms-length relationship while they collaborate more strongly with one or two major customers which generate the largest scale of business. Their longrun relation with these companies also enable them to have information about the companies' ordering or delivery behavior. The benefits of this kind of relation is mutual; the truckload carrier has a steady flow of revenue and the company saves from the setup cost 
of negotiating with different carriers, while relying on the compliance and the security of the current truckload carrier. In this setting, an LTL carrier is always an available mode of transportation. The motivation behind LTL practices in the industry is to consolidate small shipments from many shippers in settings where the revenue from small shipments alone would not cover the cost. LTL carriers work with many customers, and typically they have preannounced freight rates, not specific for each customer.

The analysis of the TL carrier's transportation pricing problem follows based on a characterization of the retailer's optimal response. Therefore, we first model and solve the retailer's replenishment problem, which is to find the order quantity that will maximize his/her expected profits considering the two modes of transportation. Due to the complex structure exhibited by the retailer's expected profit function, this analysis follows by investigating the structural properties of the profit function, which then leads to a characterization of the optimal solution. As it will be discussed in Section 3, the model and its solution can be used not only for the specific setting in this paper but it also applies to a more general class of problems.

This paper relates to two streams of research: integrated transportation and inventory replenishment, and transportation pricing. The studies in the former area explicitly take into account the transportation costs and capacities in the replenishment problem along with inventory related costs and constraints. Their focus is to find the optimal replenishment quantities to minimize the sum of transportation and inventory related costs. See, for example, Aucamp (1982), Lee (1986), Lee (1989), Tersine and Barman (1994), Çetinkaya and Lee (2002), Jaruphongsa et al. (2005), Mendoza and Ventura (2008). The papers in the latter body of research, study the pricing of transportation services within the context of fleet management. Examples include Figliozzi et al. (2007), Topaloglu and Powell (2007), Zhou and Lee (2009).

Integrated transportation and inventory replenishment has been the subject of extensive research for the last three decades. While many of the papers in this area consider the problem in a single echelon setting (e.g., Aucamp, 1982; Lee, 1986; Tersine and Barman, 1994; Shinn et al., 1996; Burwell et al., 1997; Hwang et al., 1990; Jaruphongsa et al., 2005; Mendoza and Ventura, 2008; Rieksts and Ventura, 2008; Toptal, 2009), relatively recent papers model and solve the replenishment problems of multiple echelons under transportation considerations (see Hoque and Goyal, 2000; Çetinkaya and Lee, 2002; Chan et al., 2002; Toptal et al., 2003; Toptal and Çetinkaya, 2006; Rieksts and Ventura, 2010).

We further classify the studies on integrated transportation and inventory replenishment according to the transportation cost structure considered. Aucamp (1982), Lee (1986), Çetinkaya and Lee (2002), Toptal et al. (2003), Toptal and Çetinkaya (2006) are examples of papers that assume a single mode of transportation, in the form of a truckload shipment. They use a multiple set-ups structure to model this form of shipment. We cite Russell and Krajewski (1991), Burwell et al. (1997) and Tersine and Barman (1994) as examples of studies that consider less than truckload shipment. All-weight freight discounts and incremental freight discounts are predominantly used to represent LTL carriers' pricing. In some recent studies, a combination of TL and LTL shipments is modeled. See, for example, Mendoza and Ventura (2008), Rieksts and Ventura (2008), Rieksts and Ventura (2010). These three studies allow the utilization of a TL carrier and an LTL carrier simultaneously for the shipment of the replenishment quantity.

Our modeling of transportation costs is similar to the one in these studies (i.e., Mendoza and Ventura, 2008; Rieksts and Ventura, 2008; Rieksts and Ventura, 2010). Mainly, we assume that the TL carrier charges a fixed shipping price per truck whether it is fully or partially loaded, and the LTL carrier charges a constant price for each unit shipped. Under this transportation cost structure, the retailer may get advantage of economies of scale inherent in fixed cost of each truck by loading as many quantities as possible in a truck. If the quantity to be shipped does not justify the fixed cost of an additional truck, then the retailer uses the LTL carrier. Rieksts and Ventura (2008) solve the classical Economic Order Quantity (EOQ) model assuming the availability of a TL and an LTL carrier for inbound shipments. Mendoza and Ventura (2008) extend this model to consider a general whole price structure, given by either an all-units or an incremental quantity discount schedule. Rieksts and Ventura (2010) study a two-echelon system consisting of a warehouse and a retailer, and model the transportation costs from the warehouse to the retailer allowing both TL and LTL shipments. In the current paper, we propose a generic model where the retailer's production/inventory related expected profit function is strictly concave in the order quantity and the retailer is subject to a similar transportation cost structure as in these three papers. As a specific case, we consider the single period stochastic replenishment problem (i.e., Newsboy Problem).

In contrast to the integrated inventory and replenishment models, transportation pricing is a fairly unexplored area. Figliozzi et al. (2007) study carrier pricing in dynamic vehicle routing problems by estimating the incremental cost of new service requests as they arrive dynamically. Topaloglu and Powell (2007) study the problem of finding optimal prices for different lanes at each period of a finite planning horizon. Modeling the demand in each lane as random and dependent on the price, they also decide on the locations of empty vehicles. In a more recent paper, Zhou and Lee (2009) consider two firms competing with each other to increase their profits from transportation services they provide between two locations. In this study, demand for the services of one firm in a direction is deterministic and is a function of both his/her own and the rival's price. The authors find the optimal prices of the two firms in each direction using a Bertrand duopoly model. Different from the existing papers on transportation pricing, in the current study, we explicitly model the underlying inventory replenishment problem which generates the demand and we consider the availability of two modes of transportation with different structures. Since we focus on a single retailer, there are not multiple customer locations and the routing of trucks is not an issue for the carriers.

In addition to an analytical investigation of the retailer's replenishment problem and the TL carrier's pricing problem, we present some important observations due to a numerical study. We illustrate these results over some numerical examples. Mainly, we show that the retailer's decision of whether to use the TL carrier or not, is very sensitive to TL carrier's per truck shipping price. In fact, the TL carrier, by lowering the per truck shipping price slightly, may increase his/her revenue significantly. The LTL carrier, on the other hand, has diminishing opportunity to receive any order from the retailer at larger values of $s$, given that the TL carrier optimally decides his/her per truck shipping price. Finally, we show that if the retailer and the TL carrier coordinate their decisions regarding replenishment quantity and pricing, respectively, they may achieve some extra savings which can be shared between the two parties.

The remainder of the paper is organized as follows: In Section 2, we introduce the retailer's replenishment problem and the TL carrier's pricing problem in more detail, with relevant mathematical models. Section 3 is devoted to the analysis of the retailer's replenishment problem. In Section 4, the pricing problem of the TL carrier is analyzed. This is followed by numerical results in Section 5. Finally, the conclusions of this study are summarized in Section 6 with some future research directions. 


\section{Problem definition and notation}

In this study, we consider a system that includes a retailer and two carriers. The retailer operates in a Newsboy setting. That is, he/ she makes a single replenishment decision at the beginning of a period during which he/she faces random demand. If the order quantity $Q$ is greater than the demand, then excess items are salvaged at a $\$ v /$ unit revenue. If it is less than the demand, then the retailer incurs a lost sale cost of $\$ b /$ unit. The retailer pays for the transportation of incoming materials. There are two modes of transportation, a truckload (TL) carrier and a less-than-truckload (LTL) carrier. The TL carrier charges $\$ R$ per each truck whether it is fully or partially loaded. Each truck has a capacity of carrying $P$ units and the TL carrier has ample number of trucks available. The LTL carrier charges $\$ s$ per unit where $\frac{R}{P}<s<R$. That is, for carrying a truck load of items, using the TL carrier is always less costly than using a LTL carrier. However, if the quantity to be carried is less than $R / s$, then using the LTL carrier is a better option. More explicitly, the transportation cost of the retailer for replenishing $Q$ units is given by

$C(Q)=\min \{s(Q-i P)+i R,(i+1) R\}$,

where $i$ is the number of trucks used. That is, $i P \leqslant Q<(i+1) P$ and $i \in\{0\} \cup \mathbb{Z}^{+}\left(\mathbb{Z}^{+}\right.$is the set of all positive integers). The above expression can be rewritten as

$$
C(Q)= \begin{cases}s(Q-i P)+i R, & \text { if } \quad i P \leqslant Q<\frac{R}{s}+i P, \\ (i+1) R, & \text { if } \quad \frac{R}{s}+i P \leqslant Q<(i+1) P .\end{cases}
$$

Before introducing the retailer's expected profit function and the TL carrier's pricing problem, we next summarize the notation introduced so far and that will be used in the remaining parts of the text. $r \quad$ retail price per unit

$b \quad$ retailer's per unit lost sale cost

$v \quad$ salvage value of an item unsold at the retailer

$c \quad$ procurement cost per unit $(v<c<r)$

$R \quad$ per truck shipping price that the TL carrier charges

$\bar{R} \quad$ minimum value of $R$ that the TL carrier aims for

$P \quad$ capacity of a truck in number of units

$s \quad$ per unit shipping price that the LTL carrier charges $(r>c+s)$

Q retailer's replenishment quantity

$C(Q) \quad$ transportation cost of the retailer for replenishing $Q$ units

$\Pi_{T L}(Q, R)$ TL carrier's revenue if the retailer replenishes $Q$ units at $\$ R$ per truck shipping price

$X \quad$ random variable showing demand in the single period

$f(x) \quad$ probability density function of demand

$F(x) \quad$ cumulative distribution function of demand

$\mu \quad$ expected value of demand

$G(Q) \quad$ retailer's expected profit function excluding the truck costs

$H(Q) \quad$ retailer's expected profit function

For given values of $R, P$ and $s$, retailer's expected profit for replenishing $Q$ units, i.e. $H(Q)$, is given by $G(Q)-C(Q)$ where

$G(Q)=(r-v) \mu-(c-v) Q+(r+b-v) \int_{Q}^{\infty}(Q-x) f(x) d x$.

In this paper, we consider a case where the LTL carrier's per unit shipping price $s$ is already known. We study the TL carrier's problem of finding the value of $R$ that maximizes his/her revenue from the business with the current retailer. The TL carrier incurs operating costs (e.g., oil, driver wages) related to the his/her business with the current retailer. These costs are considered within $\bar{R}$, which is the minimum shipping price per truck that the TL carrier aims for. In this setting, the TL carrier does business with the current retailer as long as he/she is not at loss. Therefore, he/she aims for at least a revenue of $\$ \bar{R}$ for each truck. We note that, if the TL carrier has a target for the minimum profit from each truck, then this value can also be incorporated in $\bar{R}$.

We assume that the operating costs and the market conditions are such that practical values of $R$ should satisfy the following inequality system: $\frac{\bar{R}}{p} \leqslant \frac{R}{p}<s<R$. If the value of $R$ that maximizes the retailer's expected profits under the constraint $R<s P$, is not greater than or equal to $\bar{R}$, then the TL carrier does not consider his/her business with the retailer as profitable. Therefore, he/she may quote a very high value of $R$ (i.e., a value of $R$ that is greater than $s P$ ) in practice as a means for implicitly rejecting the business of the retailer.

The TL carrier's revenue $\Pi_{T L}(Q R)$ in this system is given by

$\Pi_{T L}(Q, R)= \begin{cases}i \times R, & \text { if } \quad i P \leqslant Q<\frac{R}{s}+i P \\ (i+1) \times R, & \text { if } \quad \frac{R}{s}+i P \leqslant Q<(i+1) P,\end{cases}$

where $i \in\{0\} \cup \mathbb{Z}^{+}$. Expressions (1) and (3) imply that, if $i P \leqslant Q<\frac{R}{S}+i P$, then the quantities transported by the TL carrier and the LTL carrier are $i P$ and $Q-i P$, respectively. Similarly, if $\frac{R}{s}+i P \leqslant Q<(i+1) P$, then these quantities are $Q$ and 0 in the same order.

In order to write a more explicit expression for the retailer's expected profit function, we introduce the following two functions over $Q \geqslant 0$ and $j \in\{0\} \cup \mathbb{Z}^{+}$:

$H_{1}(Q, j)=G(Q)-s Q+(s P-R) j$

and

$H_{2}(Q, j)=G(Q)-j R$.

Therefore, we have

$H(Q)= \begin{cases}H_{1}(Q, i), & \text { if } \quad i P \leqslant Q<\frac{R}{s}+i P, \\ H_{2}(Q, i+1), & \text { if } \quad \frac{R}{s}+i P \leqslant Q<(i+1) P .\end{cases}$

Given the values of $R$ and $s$, the retailer solves the following problem to decide on his/her optimal replenishment quantity.

$\max H(Q)$,

s.t. $Q \geqslant 0$.

Let the optimal solution of the above problem be $Q^{*}(R, S)$. In this paper, we first analyze the structural properties of $H(Q)$ and propose a finite time exact solution procedure for finding $Q^{*}(R, s)$ given the values of $R$ and $s$. Then, we study the underlying transportation pricing problem that the TL carrier faces. Namely, given the LTL carrier's per unit transportation charge, we study the problem of the TL carrier in deciding the value of $R$, referred to as Problem $\mathcal{T} \mathcal{L}$. Throughout this analysis, we assume that the TL carrier knows the retailer's inventory related costs, and hence, predicts the response of the retailer in terms of his/her order quantity, i.e., the value of $Q^{*}(R, s)$.

The pricing problem of the TL carrier as described above can be formulated as follows:

$\mathcal{T L P}$ :

$\max \Pi_{T L}\left(Q^{*}(R, s), R\right)$,

s.t. $R \geqslant \bar{R}$,

$R<s P$.

The first constraint in the above formulation restricts the feasible values of the per truck shipping price $R$ to those real numbers that are at least equal to the minimum target revenue $\bar{R}$. It is natural to expect that the minimum target revenue $\bar{R}$ is greater than the value of the LTL carrier's per unit shipping price (i.e., $\bar{R}>s$ ). Therefore, this constraint also implies that $R>S$, and hence, the retailer would not 
use the TL carrier to ship one unit of the product at any feasible value of the per truck shipping price. The second constraint ensures that there are some order quantities of the retailer for which the TL carrier would be a less costly mode of transportation. Note that if the second constraint was not satisfied (i.e., $R \geqslant s P$ ), then the retailer would use the LTL carrier for all order quantities and would never be better off by utilizing the TL carrier.

In the next section, we study the retailer's replenishment problem to characterize his/her response to given values of $R$ and $s$. The retailer decides his/her order quantity after both the TL carrier and the LTL carrier announce their price schedules. Therefore, the retailer is subject to those values of $R$ and $s$ such that $\frac{R}{P}<s<R$.

\section{Retailer's replenishment problem}

In this section, we propose a characterization for the maximizer of $H(Q)$. As seen in Fig. 1, $H(Q)$ is a piecewise function. The proposed characterization for maximizing $H(Q)$ will follow due to some of its structural properties. We would like to note that these properties will only utilize the fact that $G(Q)$ is a strictly concave function of $Q$ (i.e., $\frac{d^{2} G(Q)}{d Q^{2}}<0$ ). Therefore, the analysis herein is not restricted to the Newsboy setting but it also applies to a more general class of problems where the production/inventory related expected profits of the retailer is strictly concave and his/her replenishment problem has a nonrecurring nature. Following the solution to the retailer's replenishment problem, we will study the transportation pricing problem of the TL carrier in the next section. We start by presenting some general properties of $H(Q)$ in the next two lemmas.

Lemma 1. $H(Q)$ is a continuous function of $Q$.

Proof. The proof is omitted as it is trivial.

As seen in Expression (6), $H(Q)$ attains values given by $H_{1}(Q, j)$ alternating with $\mathrm{H}_{2}(Q, j)$ in successive intervals of $Q$. Both of these functions are based on the production/inventory related expected profits of the retailer, i.e., $G(Q)$. Since $G(Q)$ is a strictly concave function, it follows from Expressions (4) and (5) that $H_{1}(Q, j)$ and $H_{2}(Q, j)$ are strictly concave functions over $Q$ for fixed $j$. Therefore, they have unique maximizers. Let $q^{*}$ be the maximizer of $G(Q)$. It can be observed that $q^{*}$ is also the unique maximizer of $H_{2}(Q, j), \forall j$. Similarly, let $z^{*}$ be the unique maximizer of $H_{1}(Q j), \forall j$. Expression (4) implies that $G^{\prime}\left(z^{*}\right)=s$. The following lemma characterizes the ordinal relationship between $q^{*}$ and $z^{*}$.

Lemma 2. We have $z^{*}<q^{*}$.

Proof. See Appendix A.1 for the proof.
Before presenting the development of the proposed characterization for the optimal solution, let us define the following terms: we say $z^{*}$ is realizable if there exists $i \in\{0\} \cup \mathbb{Z}^{+}$such that $i P \leqslant Z^{*}<\frac{R}{s}+i P$. Similarly, $q^{*}$ is realizable if there exists $i \in\{0\} \cup \mathbb{Z}^{+}$such that $\frac{R}{s}+i P \leqslant q^{*}<(i+1) P$.

The proposed characterization for $Q^{*}(R, s)$ will be used for solving the TL carrier's transportation pricing problem. This characterization utilizes some properties of $H(Q)$ which are dependent on the values of $z^{*}$ and $q^{*}$. These properties are presented in the next four lemmas.

Lemma 3. We have $H(Q)<H\left(z^{*}\right), \forall Q<z^{*}$.

Proof. See Appendix A.2 for the proof.

Lemma 3 implies that $z^{*}$ dominates all other quantities that are smaller than itself, therefore, in maximizing $H(Q)$, order quantities less than $z^{*}$ should not be considered. In the next lemma, we show that $q^{*}$ dominates all order quantities that are greater than itself, therefore, in maximizing $H(Q)$, order quantities greater than $q^{*}$ should not be considered.

Lemma 4. We have $H(Q)<H\left(q^{*}\right), \forall Q>q^{*}$.

Proof. The proof is similar to that of Lemma 3 and follows by considering the two cases where $q^{*}$ is either realizable or not realizable.

Lemmas 3 and 4 jointly imply that we should focus on $Q$ such that $z^{*} \leqslant Q \leqslant q^{*}$ while optimizing $H(Q)$. In Lemma 5 and 6 , by a similar analysis, we further eliminate some quantity values between $z^{*}$ and $q^{*}$.

Lemma 5. Let $Q$ be an order quantity such that $z^{*}<Q<q^{*}$ and $j P<Q<\frac{R}{s}+j P, j \in\{0\} \cup \mathbb{Z}^{+}$. Then, we have $H\left(\max \left\{z^{*}, j P\right\}\right)>H(Q)$.

Proof. See Appendix A.3 for the proof.

Lemma 6. Let $Q$ be an order quantity such that $z^{*}<Q<q^{*}$ and $\frac{R}{s}+j P \leqslant Q<(j+1) P, j \in\{0\} \cup \mathbb{Z}^{+}$. Then, we have $H\left(\min \left\{q^{*}\right.\right.$, $(j+1) P\})>H(Q)$.

Proof. The proof is omitted as it is similar to that of Lemma 5. Lemmas 5 and 6 imply that among the order quantities between $z^{*}$ and $q^{*}$, we should only consider multiples of a full truck load. The following theorem provides a characterization of the retailer's optimal order quantity.

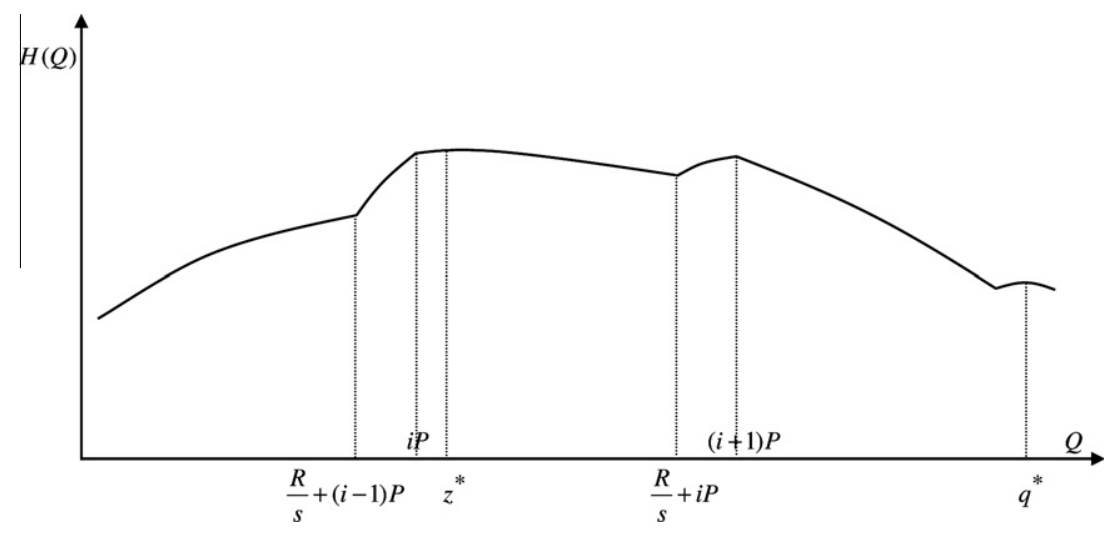

Fig. 1. An illustration of the retailer's expected profits. 
Theorem 1. The order quantity which maximizes $H(Q)$ is given by

$$
\begin{aligned}
Q^{*}(R, s)= & \arg \max \left\{H\left(z^{*}\right), H\left(q^{*}\right), H(k P) \text { s.t. } \quad k=\left\lceil\frac{z^{*}}{P}\right\rceil, \ldots,\left\lfloor\frac{q^{*}}{P}\right\rfloor,\right. \\
& \left.k \in\{0\} \cup \mathbb{Z}^{+}\right\} .
\end{aligned}
$$

Proof. The proof follows from Lemmas 3-6.

The above theorem implies that the retailer does not use the LTL carrier if both $z^{*}$ and $q^{*}$ are multiples of a full truck load. The LTL carrier would generate some revenue from the business of the retailer only if the retailer orders $z^{*}$ and $z^{*}$ is realizable, or if the retailer orders $q^{*}$ and $q^{*}$ is not realizable. Because in all other cases, the retailer uses only the TL carrier. The following lemma further shows that in the second case (i.e., $Q^{*}(R, s)=q^{*}$ and $q^{*}$ is not realizable), the quantity shipped using the LTL carrier is zero.

Lemma 7. If there exists $j, j \in\{0\} \cup \mathbb{Z}^{+}$such that $j P \leqslant q^{*}<\frac{R}{s}+j P$, that is $q^{*}$ is not realizable, then the retailer orders $q^{*}$ only if $q^{*}$ is an integer number full truck loads.

Proof. See Appendix A.4 for the proof.

In the following lemma, we show that, in the optimal solution, it is not possible for the retailer to order in an amount of $z^{*}$ and use only the TL carrier.

Lemma 8. If there exists $j, j \in\{0\} \cup \mathbb{Z}^{+}$such that $\frac{R}{s}+j P \leqslant z^{*}<$ $(j+1) P$, that is if $z^{*}$ is not realizable, then it is not optimal.

Proof. See Appendix A.5 for the proof.

The structure of the solution for maximizing $H(Q)$, as given in Theorem 1, reveals further results if the retailer operates in the Newsboy setting. Using Expression (2), it can be shown that $z^{*}$ should satisfy

$F\left(z^{*}\right)=\frac{r+b-c-s}{r+b-v}$,

and similarly, $q^{*}$ should satisfy

$F\left(q^{*}\right)=\frac{r+b-c}{r+b-v}$

The above two expressions imply that if the retail price $(r)$, lost sale cost $(b)$, or salvage value $(v)$ increases, $z^{*}$ and $q^{*}$ increase. If the procurement cost $(c)$ increases, both $z^{*}$ and $q^{*}$ decrease. However, the integer multiples of a full truck load between $z^{*}$ and $q^{*}$ may remain the same. $Q^{*}(R, s)$ is, therefore, nondecreasing in $r, b$ and $v$, and nonincreasing in $c$.

\section{An analysis of the truckload carrier's pricing problem}

In this section, we study the transportation pricing problem faced by the TL carrier. Recall that, we define the transportation pricing problem of the TL carrier as determining the value of the per truck price $R$ that maximizes the TL carrier's expected profits under the optimal response of the retailer, for a given price schedule of the LTL carrier (i.e., unit shipping price $s>0$ ). A formulation of this problem is provided in Section 2 and is referred to as Problem $\mathcal{T L P}$.

Solving the transportation pricing problem of the TL carrier exhibits certain challenges primarily due to the piecewise structure of the retailer's expected profit function. As discussed in
Section 3, there is no simple analytical expression for $Q^{*}(R, S)$. However, we have developed a characterization of the optimal solution through showing some structural properties of the objective function. In our following analysis for the TL carrier's transportation pricing problem, we will follow a similar approach. Based on our earlier results, we will further simplify the formulation of this problem, which will lead us to the optimal solution. In our analysis, we solve $\mathcal{T L P}$ for the general demand distribution. We note that, for a specific demand distribution the solution may be further simplified.

The approach that we follow in solving $\mathcal{T L P}$ utilizes the characterization of the retailer's optimal solution which we have derived in Section 3. This solution is stated in Theorem 1. It is implied by this theorem that $Q^{*}(R, s)$ can be $z^{*}$, or $q^{*}$ or any integer number of full truck loads between those quantities. Considering this implication, we divide all possible values of $R$ (all real numbers such that $R<s P$ and $R \geqslant \bar{R}$ ) into three groups and proceed our analysis thereafter. More specifically, we optimize out of all feasible $R$ values which lead to $z^{*}$. Secondly, we optimize over all feasible $R$ values which lead to an optimal replenishment quantity of the retailer given by $k P$, where $k \in\left\{\left\lceil\frac{z^{*}}{P}\right\rceil, \ldots,\left[\frac{q^{*}}{P}\right]\right\}$. Thirdly, we solve $\mathcal{T} \mathcal{P}$ over all feasible values which lead to $q^{*}$ as the optimal replenishment quantity of the retailer. Finally, among these solutions, we choose the $R$ value which results in the maximum revenue for the TL carrier. It is important to note that the convenience of using the proposed characterization for $Q^{*}(R, S)$ along with this approach is that, the possible values for the optimal solution of the retailer (i.e., $z^{*}$, or $q^{*}$ or any full truck load in between) is independent of the specific value of $R$ for fixed $s$.

In what follows, we first present Propositions $1-3$ to identify the feasible $R$ values which lead to each type of solution discussed above. Then, we formulate the three subproblems and discuss their solutions.

Proposition 1. The optimal replenishment quantity of the retailer is given by $q^{*}$ if and only if we have $H_{2}\left(q^{*},\left[\frac{q^{*}}{P}\right]\right) \geqslant H_{1}\left(z^{*},\left\lfloor\frac{Z^{*}}{P}\right\rfloor\right)$ and $H_{2}\left(q^{*},\left\lceil\frac{q^{*}}{P}\right\rceil\right) \geqslant H_{1}(k P, k), \forall k$ such that $k=\left\lceil\frac{Z^{*}}{P}\right\rceil, \ldots,\left\lfloor\frac{q^{*}}{P}\right\rfloor$.

Proof. See Appendix A.6 for the proof.

Proposition 2. The optimal replenishment quantity of the retailer is given by $z^{*}$ if and only if we have $H_{1}\left(z^{*},\left\lfloor\frac{z^{*}}{P}\right\rfloor\right) \geqslant H_{2}\left(q^{*},\left\lceil\frac{q^{*}}{P}\right\rceil\right)$ and $H_{1}\left(z^{*},\left\lfloor\frac{z^{*}}{P}\right\rfloor\right) \geqslant H_{1}(k P, k), \forall k$ such that $k=\left\lceil\frac{z^{*}}{P}\right\rceil, \ldots,\left\lfloor\frac{q^{*}}{P}\right\rfloor$.

Proof. The proof is omitted as it is similar to that of Proposition 1. $\square$

Proposition 3. The optimal replenishment quantity of the retailer is given by $k P$, for some $k \in \mathbb{Z}^{+}$and $\left\lceil\frac{z^{*}}{P}\right\rceil \leqslant k \leqslant\left\lfloor\frac{q^{*}}{P}\right\rfloor$ if and only if $H_{1}(k P, k) \geqslant H_{2}\left(q^{*},\left\lceil\frac{q^{*}}{P}\right\rceil\right), H_{1}(k P, k) \geqslant H_{1}\left(z^{*},\left\lfloor\frac{z^{*}}{P}\right\rfloor\right)$, and $H_{1}(k P, k) \geqslant$ $H_{1}(j P, j) \forall j=\left\lceil\frac{z^{*}}{P}\right\rceil, \ldots,\left\lfloor\frac{q^{*}}{P}\right\rfloor$.

Proof. See Appendix A.7 for the proof.

Let $R^{*}\left(q^{*}\right)$ be the value of $R$ which maximizes the TL carrier's expected revenue among all feasible $R$ values that lead to $q^{*}$ as the optimal replenishment quantity of the retailer. Similarly, let $R^{*}\left(z^{*}\right)$ be the value of $R$ which maximizes the TL carrier's expected revenue among all feasible $R$ values that lead to $z^{*}$ as the optimal replenishment quantity of the retailer. Finally, let $R^{*}(k P)$ be the value of $R$ which maximizes the TL carrier's expected revenue among all feasible $R$ values that lead to $k P$ as the optimal replenishment quantity of the retailer, $k$ ranging from $\left\lceil\frac{z^{*}}{P}\right\rceil$ to $\left\lfloor\frac{q^{*}}{P}\right\rfloor$. 
Note that we have by Proposition 1 that $R^{*}\left(q^{*}\right)$ is the solution to the following problem:

$\mathcal{P} 1$

$$
\begin{array}{ll}
\max & \Pi_{T L}\left(q^{*}, R\right) \\
\text { s.t. } & \left.H_{2}\left(q^{*}, \mid \frac{q^{*}}{P}\right\rceil\right) \geqslant H_{1}\left(z^{*},\left\lfloor\frac{z^{*}}{P}\right\rfloor\right), \\
& \left.H_{2}\left(q^{*},\left\lceil\frac{q^{*}}{P}\right\rceil\right) \geqslant H_{1}(k P, k), \quad k=\left\lceil\frac{z^{*}}{P}\right\rceil, \ldots, \mid \frac{q^{*}}{P}\right\rfloor, \\
& R<s P, \\
& R \geqslant \bar{R} .
\end{array}
$$

Similarly, we have from Proposition 2 that $R^{*}\left(z^{*}\right)$ is the solution to the following problem:

P2:

$$
\max \Pi_{T L}\left(z^{*}, R\right)
$$$$
\text { s.t. } H_{1}\left(z^{*},\left\lfloor\frac{z^{*}}{P}\right\rfloor\right) \geqslant H_{2}\left(q^{*},\left\lceil\frac{q^{*}}{P}\right\rceil\right) \text {, }
$$$$
H_{1}\left(z^{*},\left\lfloor\frac{Z^{*}}{P}\right\rfloor\right) \geqslant H_{1}(k P, k), \quad k=\left\lceil\frac{Z^{*}}{P}\right\rceil, \ldots,\left\lfloor\frac{q^{*}}{P}\right\rfloor,
$$$$
R<s P \text {, }
$$$$
R \geqslant \bar{R} \text {. }
$$

Finally, we have from Proposition 3 that for any integer value of $k$ such that $\left\lceil\frac{z^{*}}{P}\right\rceil \leqslant k \leqslant\left\lfloor\frac{q^{*}}{P}\right\rfloor, R^{*}(k P)$ is the solution to the following problem:

$\mathcal{P} 3$ :

$$
\max \Pi_{T L}(k P, R)
$$$$
\text { s.t. } \quad H_{1}(k P, k) \geqslant H_{2}\left(q^{*},\left\lceil\frac{q^{*}}{P}\right\rceil\right) \text {, }
$$$$
H_{1}(k P, k) \geqslant H_{1}\left(z^{*},\left\lfloor\frac{Z^{*}}{P}\right\rfloor\right) \text {, }
$$$$
H_{1}(k P, k) \geqslant H_{1}(j P, j), \quad j=\left\lceil\frac{Z^{*}}{P}\right\rceil, \ldots,\left\lfloor\frac{q^{*}}{P}\right\rfloor,
$$$$
R<s P
$$$$
R \geqslant \bar{R}
$$

The following corollary characterizes the optimal solution of Problem $\mathcal{T L P}$.

Corollary 1. The solution to $\mathcal{T L P}$ is given by the per truck cost $R$ among $R^{*}\left(q^{*}\right), R^{*}\left(z^{*}\right)$ and $R^{*}(k P) \forall k$ such that $\left\lceil\frac{z^{*}}{P}\right\rceil \leqslant k \leqslant\left|\frac{q^{*}}{P}\right|$, which gives the maximum expected revenue for the TL carrier. In other words, the solution to $\mathcal{T L P}$ is

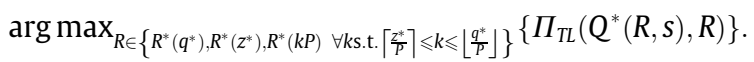

Note that, the definitions of $R^{*}\left(q^{*}\right), R^{*}\left(z^{*}\right)$ and $R^{*}(k P)$ imply that, in Expression (7), we have $Q^{*}(R, s)=q^{*}, Q^{*}(R, s)=z^{*}$ and $Q^{*}(R, s)=k P$ for $R=R^{*}\left(q^{*}\right), R=R^{*}\left(z^{*}\right)$ and $R=R^{*}(k P)$, respectively. In what follows, we provide simplified expressions for obtaining $R^{*}\left(q^{*}\right), R^{*}\left(z^{*}\right)$ and $R^{*}(k P) \forall k$ such that $\left\lceil\frac{z^{*}}{P}\right\rceil \leqslant k \leqslant\left\lfloor\frac{q^{*}}{P}\right\rfloor$ as solutions to the mathematical formulations presented earlier (i.e., $\mathcal{P} 1, \mathcal{P} 2, \mathcal{P} 3$ ). These results are presented in Lemmas 9-11.

Lemma 9. The value of $R$ which maximizes the TL carrier's expected revenue among all feasible $R$ values that lead to $q^{*}$ as an optimal replenishment quantity of the retailer, that is $R^{*}\left(q^{*}\right)$, is given by

$$
\begin{gathered}
\min \left\{\frac{G\left(q^{*}\right)-G\left(z^{*}\right)+s z^{*}-s P\left\lfloor\frac{z^{*}}{P}\right\rfloor}{\left\lceil\frac{q^{*}}{P}\right\rceil-\left\lfloor\frac{z^{*}}{P}\right\rfloor}, \frac{G\left(q^{*}\right)-G(k P)}{\left\lceil\frac{q^{*}}{P}\right\rceil-k},\right. \\
\left.\quad \forall k \text { s.t. }\left\lceil\frac{z^{*}}{P}\right\rceil \leqslant k \leqslant\left\lfloor\frac{q^{*}}{P}\right\rfloor, k P \neq q^{*}\right\}
\end{gathered}
$$

if the above function value is greater than or equal to $\bar{R}$. Otherwise, there is no value of $R$ that leads to $q^{*}$ as the optimal replenishment quantity of the retailer while satisfying the TL carrier's target revenue constraint.

Proof. See Appendix A.8 for the proof.

The proof of the above lemma implies that in the expression for $R^{*}\left(q^{*}\right)$, if the minimum is given by the first term, then the first constraint in $\mathcal{P} 1$ is satisfied at equality, i.e., $H_{2}\left(q^{*},\left\lceil\frac{q^{*}}{\mathcal{P}}\right\rceil\right)=H_{1}\left(z^{*},\left\lfloor\frac{z^{*}}{\mathcal{P}}\right\rfloor\right)$. In this case, both $q^{*}$ and $z^{*}$ maximize the retailer's expected profits. Likewise, if the minimum is given by the second term for some $\left.k \in\left\{\left\lceil\frac{Z^{*}}{P}\right\rceil, \ldots, \mid \frac{q^{*}}{P}\right\rfloor\right\}$, then the second constraint in $\mathcal{P} 1$ is satisfied at equality, i.e., $H_{2}\left(q^{*},\left\lceil\frac{q^{*}}{P}\right\rceil\right)=H_{1}(k P, k)$, which implies that both $q^{*}$ and $k P$ maximize the retailer's expected profits. In this case, the TL carrier may need to give the retailer additional incentive for him to choose $q^{*}$ such as offering a per truck cost that is slightly lower than $R^{*}\left(q^{*}\right)$.

Lemma 10. The value of $R$ which maximizes the TL carrier's expected revenue among all feasible $R$ values that lead to $z^{*}$ as the optimal replenishment quantity of the retailer, that is $R^{*}\left(z^{*}\right)$, is given by $S P-\varepsilon$ ( $\varepsilon$ is a positive, very small number), if

$$
\begin{aligned}
& \max \left\{\bar{R}, \frac{G\left(q^{*}\right)-G\left(z^{*}\right)+s z^{*}-s P\left\lfloor\frac{z^{*}}{P}\right\rfloor}{\left\lceil\frac{q^{*}}{P}\right\rceil-\left\lfloor\frac{z^{*}}{P}\right\rfloor}, \frac{G(k P)-G\left(z^{*}\right)+s z^{*}-s P\left\lfloor\frac{z^{*}}{P}\right\rfloor}{k-\left\lfloor\frac{Z^{*}}{P}\right\rfloor},\right. \\
& \left.\forall k \text { s.t. }\left\lceil\frac{z^{*}}{P}\right\rceil \leqslant k \leqslant\left\lfloor\frac{q^{*}}{P}\right\rfloor, k P \neq z^{*}\right\}
\end{aligned}
$$

is less than sP. Otherwise, there is no value of $R$ that leads to $z^{*}$ as the optimal replenishment quantity of the retailer while satisfying the TL carrier's target revenue constraint.

Proof. See Appendix A.9 for the proof.

We would like to note that Lemma 10 originates from mathematical formulation $\mathcal{P} 2$, and the terms of Expression (8) are lower bounds on $R$ based on evaluating the first, second and the fourth constraints of $\mathcal{P} 2$. The retailer may order $z^{*}$ if these constraints are satisfied, however, if the third constraint is violated (i.e., $R \geqslant s P$ ), then the retailer utilizes only the LTL carrier. Hence, the TL carrier can increase his/her price to charge per truck up to $s P$, however he/she should cut the price slightly, that is by $\varepsilon$, to gain the business of the retailer. The value of $s P-\varepsilon$ in this case, should not be smaller than the lower bounds mentioned above.

Lemma 11. The value of $R$ which maximizes the TL carrier's expected revenue among all feasible $R$ values that lead to $k P$ ( $k$ is an integer such that $\left\lceil\frac{z^{*}}{P}\right\rceil \leqslant k \leqslant\left\lfloor\frac{q^{*}}{P}\right\rfloor$ ) as an optimal replenishment quantity of the retailer, that is $R^{*}(k P)$, is given by

$\min \left\{A, s P-\varepsilon, \frac{G(k P)-G(j P)}{k-j}, \quad \forall j\right.$ s.t. $\left.\left\lceil\frac{z^{*}}{P}\right\rceil \leqslant j \leqslant k-1\right\}$,

where $\varepsilon$ is a positive, very small number, and

$A= \begin{cases}\infty, & \text { if } z^{*}=k P, \\ \frac{G(k P)-G\left(z^{*}\right)+S z^{*}-s P\left\lfloor\frac{Z^{*}}{P}\right\rfloor}{k-\left\lfloor\frac{Z^{*}}{P}\right\rfloor}, & \text { o.w. }\end{cases}$

if the value given by Expression (9) is greater than or equal to

$\max \left\{B, \bar{R}, \frac{G(j P)-G(k P)}{j-k}, \forall j\right.$ s.t. $\left.j=(k+1), \ldots,\left\lfloor\frac{q^{*}}{P}\right\rfloor\right\}$, 
where

$B= \begin{cases}0, & \text { if } q^{*}=k P, \\ \frac{G\left(q^{*}\right)-G(k P)}{\left\lceil\frac{q^{*}}{P}\right\rceil-k}, & \text { o.w. },\end{cases}$

Otherwise, there is no value of $R$ that leads to $k P$ as the optimal replenishment quantity of the retailer while satisfying the TL carrier's target revenue constraint.

Proof. See Appendix A.10 for the proof.

Note again that Lemma 11 originates from mathematical formulation $\mathcal{P} 3$, and the terms of Expression (10) are lower bounds on $R$ based on evaluating the first, third and the fifth constraints of $\mathcal{P} 3$. The value of $s P-\varepsilon$ in Expression (9), should not be smaller than these lower bounds.

\section{Numerical illustrations}

In this section, we present five numerical examples for which the retailer's replenishment and the TL carrier's pricing problem are solved. Through these examples, we illustrate how the proposed solutions in the paper can be applied and some insights we gained over a more extensive numerical analysis.

Example 1. Consider a setting where $c=12, r=32, v=11, b=14$, $X \approx U(0,1000$ ) (demand is uniformly distributed between 0 and 1000 ), the LTL carrier's per unit shipping price $s$ is 4 , the TL carrier owns trucks of capacity 307 and aims to earn a minimum revenue of 100 per truck.

For the above example, let us consider the TL carrier's pricing problem. The retailer's expected profit function without the transportation costs, i.e. $G(Q)$, is $-0.0175 Q^{2}+34 Q-7000$. It turns out that $q^{*}=971.43$ and $z^{*}=857.143$. Lemma 9 implies that there is no value of $R$ greater than $\bar{R}$ that would lead to $q^{*}$ as an optimal order quantity of the retailer. Using Lemmas 10 and 11 , we have $R^{*}\left(z^{*}\right)=1227.99, R^{*}(921)=1156.64$. Corollary 1 further leads to the fact that $\Pi_{T L}(857.143,1227.99)=2455.98$ and $\Pi_{T L}(921,1156$. $64)=3469.92$. The TL carrier's revenue is maximized when $R=1156.64$ and the retailer uses the TL carrier for inbound transportation. However, at $R=1156.64$, the retailer is indifferent between ordering $z^{*}=857.143$ and $3 \times P=921$. At both quantities, the retailer's expected profit is 5999.86. Note that, if the retailer chooses to order $z^{*}$, then he/she rents two trucks from the TL carrier to ship 614 units and uses the LTL carrier for transporting the remaining amount of 243.143. If he/she chooses to order 921 units, it is optimal for the retailer to ship this amount through the TL carrier utilizing three trucks. In this case, the retailer would probably choose to order 921 units for the practical convenience of doing business with one carrier. Nevertheless, if the TL carrier wants to implicitly force the retailer to order 921 units, he/she may quote a slightly lower price than 1156.64 per truck. For example, if the TL carrier sets $R=1156$, then $H\left(z^{*}\right)=6001.14$ and $H(921)=6001.78$, therefore, the retailer's optimal order quantity is 921 and he/she uses only the TL carrier. This leads to a revenue of 3468 for the TL carrier.
Example 2. Consider a setting where $c=16, r=32, v=11, b=14$, $X \approx U(0,1000$ ) (demand is uniformly distributed between 0 and 1000 ), the TL carrier owns trucks of capacity 250 and aims to earn a minimum revenue of 150 per truck. The following values for the LTL carrier's per unit shipping price $s$ will be considered: $1.5,2,2.5$, 3, 3.5, 4 and 4.5 .

For each value of $s$, Table 1 presents the TL carrier's optimal pricing decision in terms of per truck shipping price $R$, the retailer's order quantity, and the quantities shipped by the TL carrier and the LTL carrier. The results imply that the TL carrier has a greater opportunity to increase his/her profits through pricing at larger values of $s$. Furthermore, we observe that at larger values of $s$, the LTL carrier has less chance of being utilized by the retailer, given that the TL carrier solves his/her pricing problem optimally. This may sound quite intuitive at first, however, consider the case where $s=4.5$ and assume that the truckload carrier sets $R=1118$, i.e., he/she does not decide $R$ optimally. In this case, the retailer would order a quantity of 728.57 while shipping 228.57 of this amount using the LTL carrier. Instead, by deciding $R$ optimally, the TL carrier extracts better gainings, which further leads to no usage of the LTL carrier. At smaller values of $s$, even if the TL carrier makes his/her pricing decision optimally, the retailer may use the LTL carrier for shipping some amount.

Example 3. Consider a setting where $c=15, r=30, v=11, b=13$, and exponentially distributed demand with rate $\lambda=0.002$. The TL carrier owns trucks of capacity 200 and aims to earn a minimum revenue of 100 per truck. The LTL carrier's per unit shipping price $s$ is 2 .

In this example, the retailer's expected profit function without transportation costs, i.e. $G(Q)$, is $9500-4 Q-16000 e^{-0.002 Q}$. Corollary 1 implies that the TL carrier's optimal value of per truck shipping price is 399.99. At this value of $R$, the retailer orders $z^{*}=836.988$. This results in an expected profit of 1478.075 for the retailer and a revenue of 1599.996 for the TL carrier. Setting $R$ to 399.99 maximizes the TL carrier's revenues, however, there exist other values of $R$ which lead to better gainings for the retailer and TL carrier jointly. For example, if the TL carrier agrees to charge 256.564 for each truck and the retailer orders $Q=1000$ units, then the retailer's expected profits increase to 2051.812. In this case, the TL carrier's revenue reduces to 1282.82 . Since the retailer's gain (i.e., 573.737) exceeds the TL carrier's loss (317.176), the retailer may compensate the TL carrier for the losses and still be better off. This may lead to a win-win solution for both the parties.

We note that, $R$ and $Q$ values that improve the joint benefits of the TL carrier and the retailer can be found in the following way: First, we form the set of $R$ and $Q$ pairs where $Q$ assumes possible values for the retailer's optimal order quantity and $R$ maximizes the TL carrier's revenue while leading to $Q$ as the retailer's expected profit maximizer. Theorem 1, Lemmas 9-11 imply that this set is finite and countable. Then, we evaluate these pairs to find the one that maximizes the joint benefits of the parties. In the above example, the possible values for the retailer's optimal order quantity are $z^{*}=836.988, q^{*}=1039.72$ and $Q=1000$. It follows from Lemma 9 that there is no value of $R$ greater than or equal to 100

Table 1

Quantities shipped by the two carriers as a result of TL carrier's optimal pricing decision for varying values of $s$.

\begin{tabular}{|c|c|c|c|c|c|}
\hline$s$ & TL carrier's optimal $R$ & Retailer's order quantity & Quantity shipped by the TL carrier & \# Trucks utilized & Quantity shipped by the LTL carrier \\
\hline 1.5 & 374.999 & 814.286 & 750 & 3 & 64.286 \\
\hline 2 & 499.999 & 800 & 750 & 3 & 50 \\
\hline 2.5 & 624.999 & 785.714 & 750 & 3 & 35.714 \\
\hline 3 & 749.999 & 771.429 & 750 & 3 & 21.429 \\
\hline 3.5 & 874.999 & 757.143 & 750 & 3 & 7.143 \\
\hline 4 & 999.107 & 750 & 750 & 3 & 0 \\
\hline 4.5 & 1116.964 & 750 & 750 & 3 & 0 \\
\hline
\end{tabular}


Table 2

Quantities shipped and the benefits for the parties resulting from the retailer's optimal replenishment decision for varying values of $s$.

\begin{tabular}{llllll}
\hline$s$ & $\begin{array}{l}\text { Retailer's order } \\
\text { quantity }\end{array}$ & $\begin{array}{l}\text { Quantity shipped by the } \\
\text { LTL carrier }\end{array}$ & $\begin{array}{l}\text { Quantity shipped by the } \\
\text { TL carrier }\end{array}$ & $\begin{array}{l}\text { LTL carrier's } \\
\text { revenue }\end{array}$ & $\begin{array}{l}\text { TL carrier's } \\
\text { revenue }\end{array}$ \\
\hline 1.2 & 822.857 & 72.857 & 750 & 87.429 & 540 \\
profits
\end{tabular}

that would lead to $q^{*}=1039.72$ as the retailer's expected profit maximizer. We have from Lemma 10 and Lemma 11 that $R^{*}\left(z^{*}\right)=399.99$ and $R^{*}(1000)=256.564$, respectively. Therefore, we compare the pair of $R=399.99$ and $Q=836.988$ with the pair of $R=256.564$ and $Q=1000$. We choose the latter solution as the joint benefits in this case are more.

Example 1 shows that the TL carrier's revenue is very sensitive to changes in the value of $R$, mainly due to the piecewise structure of the retailer's expected profit function and the discontinuity of the TL carrier's revenue function. These characteristics of the replenishment problem and the transportation pricing problem may lead to a significant impact on the TL carrier's revenue if he/ she does not have correct information about the parameters of the retailer. The next example illustrates this assuming the TL carrier incorrectly estimates the parameters of the retailer's demand distribution.

Example 4. Consider a setting with the same parameters as of Example 3. Assume that the TL carrier has incorrect information about $\lambda$, which in fact is equal to 0.002 . Consider the following cases where the TL carrier thinks that $\lambda=0.0016, \lambda=0.0019$, or $\lambda=0.0024$.

The TL carrier finds the optimal per truck price as 399.999 , 325.085 and 330.181 if he/she does his/her computations according to $\lambda=0.0016, \lambda=0.0019$, and $\lambda=0.0024$, respectively. As the retailer's true $\lambda$ is 0.002 , the retailer decides to order 836.988 units in each case. Therefore, the TL carrier attains a revenue of 1599.996 , 1300.34, and 1320.724 under $\lambda=0.0016, \lambda=0.0019$, and $\lambda=0.0024$, respectively. We know from Example 3 that if the TL carrier had correct information about $\lambda$, he/she would set $R$ to 399.999, in which case his/her revenue would be 1599.996 . Although $\lambda=0.0019$ is closer to the true value of $\lambda=0.002$, the TL carrier's loss due to having incorrect information in this case, is more than it is in the other two cases (i.e., $\lambda=0.0016$ and $\lambda=0.0024)$. The TL carrier does not have any loss even if he/she thinks that $\lambda=0.0016$ as the optimal values of $R$ when $\lambda$ is taken as 0.0016 or 0.002 , are the same. However, in cases of $\lambda=0.0019$ and $\lambda=0.0024$, he/she ends up with respectively $18.728 \%$ and $17.454 \%$ lower revenue than what he/she would have if $\lambda$ was estimated correctly. This example illustrates the significance of accurate estimation of the retailer's parameters by the TL carrier for his/her pricing decisions.

In this paper, our focus has been to solve the pricing problem of the TL carrier in a setting where the LTL carrier's pricing scheme is known. In the next example, we consider another situation in which the LTL carrier has to make a pricing decision given the TL carrier's per truck shipping price. We show that in this operating environment, the LTL carrier can also achieve significant savings by carefully deciding the per unit shipping price.
Example 5. Consider a setting with the same parameters as of Example 2. Assume that $R=180$ and the LTL aims to earn a minimum revenue of 1.2 per unit he/she ships.

Table 2 presents the quantities shipped by the two carriers, their revenues, and the retailer's order quantity and expected profits at different values of $s$. The LTL carrier maximizes his/her revenue if the per unit shipping price is set to 1.875 . The retailer does not use the services of the LTL carrier if $s>2.5406$. Table 2 also shows the impact of carefully deciding the values of $s$ on the LTL carrier's revenue. For example, instead of setting the value of $s$ to 2.5 , the LTL carrier can increase his/her revenue by $12.5 \%$ by setting the value of $s$ to 1.875 .

It should be pointed out that, in the numerical examples of this section, demand was chosen to be either exponentially or uniformly distributed to avoid superfluous computational burden. The challenge in doing the computations manually in case of many demand distributions lies in finding the value of the integral term in Expression (2). Note that, $\int_{Q}^{\infty}(x-Q) f(x) d x$ refers to the expected amount of shortages and it appears in many inventory models with stochastic demand. Other distributions could be used to model demand, including normal distribution, gamma distribution, Laplace distribution, etc. We refer to Silver et al. (1998, p. 273), for a review of articles on different demand distributions.

\section{Conclusions and future research}

This paper analyzes the replenishment problem of a retailer and the transportation pricing problem of a truckload carrier under the presence of two carriers (one truck-load carrier and one less-thantruckload carrier). In this setting, the retailer makes a single replenishment decision at the beginning of the selling period during which she/he faces random demand. The inbound replenishment quantity can be shipped utilizing both the TL carrier and the LTL carrier. The costs due to inbound transportation are incurred by the retailer. The TL carrier charges a fixed cost for each truck whether it is fully or partially loaded. A unit shipping price is charged by the LTL carrier. The retailer's production/inventory related expected profit function is assumed to be strictly concave. This generic structure makes the results herein applicable to other problem settings as well.

An important application area of this study can be in outsourcing decisions. The costs that the retailer incurs due to TL shipments in this paper, is also known as the multiple set-ups cost structure in the literature. It can also be used to model the production setup costs of a manufacturer who incurs a fixed setup cost for operating a capacitated machine with the capability of processing several items at a time. The unit shipping price that the LTL carrier charges may be considered as the cost per unit if the manufacturer decides 
to outsource the items rather than manufacture inhouse. As a result of maximizing Expression (6), the manufacturer can end up with one of the following decisions in addition to determining the production lot size: manufacture all items inhouse, outsource all items from a third party or a combination of both.

Transportation pricing is a relatively new area in supply chain management. To the best of our knowledge, there are few studies in this area and they all study the problem within the context of fleet management. These studies take demand as exogenously given or as a simple function of price. In our study, we focus on a single customer location (i.e., a single retailer) and we explicitly model the underlying inventory replenishment problem which generates the demand. The transportation pricing problem is studied for the TL carrier modeling him/her as a revenue maximizer. A minimum target revenue per truck is assumed. This value (i.e., $\bar{R}$ ) can also be associated with the TL carrier's cost per truck when he/she serves the inbound transportation of the current retailer. The challenge behind solving the TL carrier's pricing problem as defined in this study is the due to the piecewise structure of the retailer's expected profit function. However, based on a detailed analysis of the retailer's optimization problem, the TL carrier's transportation pricing problem is solved exactly.

A notable generalization of this problem is when there are multiple LTL carriers and/or multiple TL carriers. Under the consideration that the current TL carrier has prior information about the shipping prices of all other carriers, a similar approach can be followed for formulating and solving the retailer's replenishment problem and the TL carrier's pricing problem. In some cases, this can be achieved with small modifications. For example, if all the LTL carriers have the same pricing structure (i.e., charges a fixed price per unit) and the TL carriers have identical trucks (i.e., same capacity), the solution to the retailer's replenishment problem can be updated by replacing $s$ and $R$ with the minimum of the per unit shipping prices and the minimum of the per truck shipping prices, respectively. An adaptation of the proposed solution methodology for the TL carrier's pricing problem herein can also be followed in this new setting. In order for the retailer to choose the current TL carrier instead of the other TL carriers, the current TL carrier should quote a better per truck shipping price. Therefore, the minimum of the per truck shipping prices of all other TL carriers should be treated as an upper bound on $R$.

It is worthwhile to note that, although this paper considers the case where the LTL carrier has preannounced freight rates, there may be practical situations in which the LTL carrier has to make a pricing decision given the TL carrier's per truck shipping price. Example 3 shows that there is significant potential for the LTL carrier to increase his/her revenues through pricing. We address this problem in our current research.

Numerical results show that, by coordinating the transportation pricing decision and the replenishment decision, the retailer and the TL carrier may increase their gainings. This observation leads to design of contractual agreements in this setting as a future research direction. The objectives of this paper can also be extended to other settings involving different characteristics of demand, selling period, number of replenishment opportunities, modes of carrier, transportation price structures, etc.

\section{Appendix A}

Supplementary data associated with this article can be found in the online version.

\section{Appendix B. Supplementary data}

Supplementary data associated with this article can be found, in the online version, at doi:10.1016/j.ejor.2011.05.005.

\section{References}

Aucamp, D.C., 1982. Nonlinear freight costs in the EOQ model. European Journal of Operational Research 9, 61-63.

Bureau of Transportation Statistics. 2009. National Transportation Statistics. <http://www.bts.gov> (accessed November 2009).

Burwell, T.H., Dave, D.S., Fitzpatrick, K.E., Roy, M.R., 1997. Economic lot size model for price-dependent demand under quantity and freight discounts. International Journal of Production Economics 48, 141-155.

Çetinkaya, S., Lee, C.-Y., 2002. Optimal outbound dispatch policies modeling inventory and cargo capacity. Naval Research Logistics 49, 531-556.

Chan, L.M.A., Muriel, A., Shen, Z.-J., Simchi-Levi, D., Teo, C.-P., 2002. Effective zero inventory ordering polices for the single-warehouse multi-retailer problem with piecewise linear cost structures. Management Science 48, 1446-1460.

European Commission Transport. 2006. Keep Europe Moving - Sustainable Mobility for Our Continent - Midterm Review of the European Commission's 2001 Transport White Paper. <http://ec.europa.eu/transport/strategies/ 2006_keep_europe_moving_en.htm> (accessed November 2009).

Figliozzi, M.A., Mahmassani, H.S., Jaillet, P., 2007. Pricing in dynamic vehicle routing problems. Transportation Science 41, 302-318.

Hoque, M.A., Goyal, S.K., 2000. An optimal policy for single-vendor single-buyer integrated production-inventory system with capacity constraint of transport equipment. International Journal of Production Economics 65, 305-315.

Hwang, H., Moon, D.H., Shinn, S.W., 1990. An EOQ model with quantity discounts for both purchasing price and freight cost. Computers and Operations Research $17,73-78$.

Jaruphongsa, W., Çetinkaya, S., Lee, C.-Y., 2005. A dynamic lot-sizing model with multi-model replenishments: Polynomical algorithms for special cases with dual and multiple modes. IIE Transactions 37, 453-467.

Lee, C.-Y., 1986. The economic order quantity for freight discount costs. IIE Transactions 18, 318-320.

Lee, C.-Y., 1989. A solution to the multiple set-up problem with dynamic demand. IIE Transactions 21, 266-270.

Mendoza, A., Ventura, J.A., 2008. Incorporating quantity discounts to the EOQ model with transportation costs. International Journal of Production Economics 113, 754-765.

Rieksts, B.Q., Ventura, J.A., 2008. Optimal inventory policies with two modes of freight transportation. European Journal of Operational Research 186, 576585.

Rieksts, B.Q., Ventura, J.A., 2010. Two-stage inventory models with a bi-modal transportation cost. Computers and Operations Research 37, 20-31.

Russell, R.M., Krajewski, L.J., 1991. Optimal purchase and transportation cost lot sizing for a single item. Decision Sciences 22, 940-952.

Shinn, S.W., Hwang, H., Park, S.S., 1996. Joint price and lot size determination under conditions of permissible delay in payments and quantity discounts for freight cost. European Journal of Operational Research 91, 528-542.

Silver, E.A., Pyke, D.F., Peterson, R., 1998. Inventory Management and Production Planning and Scheduling, 3rd ed. John Wiley \& Sons, New York, Chapter 7.

Tersine, R.J., Barman, S., 1994. Optimal lot sizes for unit and shipping discount situations. IIE Transactions 26, 97-101.

Topaloglu, H., Powell, W., 2007. Incorporating pricing decisions into stochastic dynamic fleet management problem. Transportation Science 41, 281-301.

Toptal, A., Çetinkaya, S., Lee, C.-Y., 2003. The buyer-vendor coordination problem: Modeling inbound and outbound cargo capacity and costs. IIE Transactions on Logistics and Scheduling 35, 987-1002.

Toptal, A., Çetinkaya, S., 2006. Contractual agreements for coordination and vendormanaged delivery under explicit transportation considerations. Naval Research Logistics 53, 1-21.

Toptal, A., 2009. Replenishment decisions under an all-units discount schedule and stepwise freight costs. European Journal of Operational Research 198, 504-510.

Zhou, W.-H., Lee, C.-Y., 2009. Pricing and competition in a transportation market with empty equipment repositioning. Transportation Research B 43, 677-691. 